\title{
Riemann-Hilbert approach and N-soliton solutions for a new four-component nonlinear Schrödinger equation
}

\author{
Xin-Mei Zhou ${ }^{1}$, Shou-Fu Tian ${ }^{1}$, Jin-Jie Yang ${ }^{1}$, and Jin-Jin Mao ${ }^{1}$ \\ ${ }^{1}$ China University of Mining and Technology
}

May 5, 2020

\begin{abstract}
A new four-component nonlinear Schrödinger equation is first proposed in this work and studied by Riemann-Hilbert approach. Firstly, we derive a Lax pair associated with a $\$ 5 \backslash$ times $5 \$$ matrix spectral problem for the four-component nonlinear Schrödinger equation. Then based on the Lax pair, we analyze the spectral problem and the analytical properties of the Jost functions, from which the Riemann-Hilbert problem of the equation is successfully established. Moreover, we obtain the $\$ \mathrm{~N} \$$-soliton solutions of the equation by solving the Riemann-Hilbert problem without reflection. Finally, we derive two special cases of the solutions to the equation for $\$ N=1 \$$ and $\$ \mathrm{~N}=2 \$$, and the local structure and dynamic behavior of the one-and two-soliton solutions are analyzed graphically.
\end{abstract}

\section{Hosted file}

FCNLS-MMAS.pdf available at https://authorea.com/users/302438/articles/432522-riemannhilbert-approach-and-n-soliton-solutions-for-a-new-four-component-nonlinear-schr\%C3\%

B6dinger-equation 